\title{
The coping strategies of community pharmacists and pharmaceutical services provided during COVID-19 in Malaysia
}

\author{
Kok Pim Kua ${ }^{1}$ and Shaun Lee ${ }^{2}$ \\ ${ }^{1}$ Puchong Health Clinic (Petaling District Health Office) \\ ${ }^{2}$ Monash University Malaysia
}

October 28, 2021

\begin{abstract}
Background The coronavirus disease 2019 (COVID-19) pandemic has resulted in profound health challenges across the globe. Pharmacists' readiness to cope with the pandemic is critical in supporting and sustaining the healthcare workforce to meet the challenges.

Objective This study aims to examine community pharmacists' views on their work environment, policies, and preparedness for safe retail patronage to prevent the transmission of COVID-19 and assess the variables influencing coping strategies during the pandemic.

Setting An online survey of pharmacists practicing in community pharmacy setting in Malaysia.

Method A questionnaire-based, cross-sectional study was conducted in Malaysia to evaluate coping strategies of community pharmacists and pharmaceutical services provided during COVID-19 pandemic. Between May 1 and July 31, 2020, the questionnaire was distributed to pharmacists working in community setting nationwide utilizing a snowball sampling method.

Main outcome measure Community pharmacists' perceptions on safety, resilience, organizational support, and pharmaceutical services offered during COVID-19 pandemic.

Results A total of 217 pharmacists participated in the study. The vast majority of community pharmacists reported a positive outlook and were able to balance working with self-care during this period. Most reported to have access to personal protective equipment such as gloves and hand sanitizers. A large proportion of community pharmacies also installed physical barriers in doorways or in front of the counter, put markings on the ground to section areas to ensure physical distancing, and controlled the number of customers who could access the pharmacy during this period. Innovations reported to be implemented included teleconsultations and providing curb-side or drive-through delivery and pickup services.
\end{abstract}

Conclusion The findings suggest the frontline and essential roles of community pharmacists in delivering pandemic responses, creating the opportunity to determine areas where community pharmacy services can be incorporated to strengthen the public health system and improve patient health outcomes.

\section{Hosted file}

Table 1.docx available at https://authorea.com/users/369365/articles/488238-the-copingstrategies-of-community-pharmacists-and-pharmaceutical-services-provided-during-covid19-in-malaysia

\section{Hosted file}

Table 2.docx available at https://authorea.com/users/369365/articles/488238-the-copingstrategies-of-community-pharmacists-and-pharmaceutical-services-provided-during-covid19-in-malaysia

\section{Hosted file}


Table 3.docx available at https://authorea.com/users/369365/articles/488238-the-copingstrategies-of-community-pharmacists-and-pharmaceutical-services-provided-during-covid19-in-malaysia

\section{Hosted file}

Table 4.docx available at https://authorea.com/users/369365/articles/488238-the-copingstrategies-of-community-pharmacists-and-pharmaceutical-services-provided-during-covid19-in-malaysia

\section{Hosted file}

Table 5.docx available at https://authorea.com/users/369365/articles/488238-the-copingstrategies-of-community-pharmacists-and-pharmaceutical-services-provided-during-covid19-in-malaysia

\section{Hosted file}

Appendix (Questionnaire).docx available at https://authorea.com/users/369365/articles/488238the-coping-strategies-of-community-pharmacists-and-pharmaceutical-services-providedduring-covid-19-in-malaysia

\section{Keywords}

Pandemic response, outbreak response, emergency response, community pharmacists, coronavirus, responding to COVID-19, safety, resilience, organizational support, pharmaceutical services, pharmacists' perceptions, healthcare, questionnaire, cross-sectional survey.

\section{Impact on practice statements}

- Community pharmacists can play a significant public health role in infectious disease mitigation.

- The COVID-19 pandemic demonstrates key functions of community pharmacists in continuity of provision of medications, medication management, preventive healthcare services, chronic and acute disease management, and referral to care.

- Important predictors for coronavirus response include age, gender, and occupational role of pharmacists.

- Telehealth services provided through community pharmacies can transform practice and patient care.

- Future outbreak preparedness strategy should develop collaborations between community pharmacists and physicians.

\section{Main text}

\section{Introduction}

Pharmacists are trusted members of multidisciplinary healthcare teams and routinely encounter patients at community pharmacies. ${ }^{1}$ The coronavirus disease 2019 (COVID-19) pandemic has led to transformation and optimization of pharmaceutical services. As one of the most highly accessible healthcare professionals, community pharmacists are often the first point of contact in many health systems for the local communities who require health-related advice on COVID- $19 .^{2}$ In tandem with physicians' practices rescheduling planned appointments for non-essential care, community pharmacies have now become the sole entry points to the primary healthcare services in many countries. Hence, it is essential that community pharmacists possess sufficient knowledge and information regarding COVID-19 to advise their patients, respond to enquiries, and be proactive in executing interventions to prevent the transmission of COVID-19 in communities.

In contrast to large healthcare facilities, community pharmacies do not have periodical, rigorous cleaning and disinfection to reduce environmental contamination. The tasks are inevitably undertaken by clinical staff (pharmacist), non-clinical staff (pharmacy assistants and dispensary technicians), or pharmacy management team. The availability of environmental sanitation and disinfection measures and practice of meticulous hand 
hygiene with readily available hand hygiene products would mitigate the risk of infection and provide some warranted reassurance of safety to both healthcare workers and patients. In addition, it is vital to evaluate the risk of occupational exposure to COVID-19 and provide staff with the necessary personal protective equipment such as masks, gloves, gowns, or goggles since physical distancing is often difficult to implement due to the relatively small spaces. This necessitates considerable caution, for instance, maintenance of safe physical distances between customers or staff, wearing of face masks by both customers and staff members, and frequent use of alcohol-based hand sanitizers in the community pharmacy compound. Improvement of public health requires effective leadership from the pharmacists who are central in providing rapid response, good coordination, and evidence-based approach that are well communicated between team members. ${ }^{3}$

\section{Aim of the study}

While there have been several studies assessing the community pharmacies response to COVID-19, only few studies have investigated the response of private community pharmacies in Asia. ${ }^{4-6}$ The objective of this study was to examine the community pharmacists' views on their work environment and policies, perceived risk and health, preparedness for safe retail patronage environment to prevent the transmission of infection, in addition to services and innovations offered during this period. The correlations between demographic characteristics of community pharmacists and response to COVID-19 crisis were also evaluated.

\section{Ethics approval}

The study was approved by the Monash Human Research Ethics Committee (Project ID: 25023).

\section{Method}

\section{Study design and study period}

This was a cross-sectional online survey conducted from May 1 until July 31, 2020 among registered community pharmacists practicing in Malaysia. Pharmacists who were working in community pharmacies were recruited. The survey comprised 43 questions formulated based upon guidance from the International Pharmaceutical Federation, ${ }^{7}$ Ministry of Health of Malaysia, Malaysian Pharmacists Society, ${ }^{8}$ websites from medical societies as well as the authors' personal experience on community pharmacy practice. The questionnaire was subsequently reviewed by two pharmacy academics and was pilot tested among 10 community pharmacists for clarity and ease of administration. The questionnaire was composed of four sections, including (1) services offered to the communities during COVID-19 pandemic; (2) availability of safety measures at workplace; (3) staff resilience; and (4) organizational support received during the pandemic (Supplementary Material). A sum score for the final three domains was computed, with higher scores indicating better support, resilience, and ability to stay healthy in the COVID-19 period.

\section{Survey administration}

An invitation email together with the survey link to the online survey platform Qualtrics was sent to recruit practicing pharmacist through the Malaysian Pharmacists Society and the authors' personal contacts. Invitations were also sent via informal pharmacist-only groups on the social media platforms (i.e. Facebook and Twitter), which were sharable to facilitate snowball sampling. In this approach, participants assisted in the recruitment of respondents through sharing of the survey among their acquaintances which increased the representativeness of participants. We also emailed the survey to pharmacy chains and stakeholders to recommend the study to their acquaintances to increase the sample's diversity. A total of three reminder emails were sent to potential participants every fortnight to increase participation rates. To prevent multiple responses from the same participants, we enabled the "Prevent Ballot Box Stuffing" option which only allowed for a single response from a respondent. Participants were provided with an explanatory statement describing the purpose of study, the risk and benefits, and that minimal personal information was collected. All participants provided informed consent electronically before they began the survey. Returned questionnaires were subsequently checked for completeness and had to have at least $50 \%$ of questions completed to be included in the final analysis. 


\section{Statistical analysis}

There were an estimated 2,000 community pharmacies that were operational in Malaysia. Assuming that there were a total of 4,000 community pharmacists working in community pharmacies, we estimated that we needed 187 responses in order to achieve a $95 \%$ confidence level and $7 \%$ confidence that these responses were representative of all pharmacists. Results were presented in frequencies, proportions, means, and standard deviations. Since not all participants completed the full questionnaire, data were presented based upon total responses received for each question. No data manipulation was performed. To investigate the associations between socio-demographic profile of the participants and outcomes of level of agreement, we performed a bivariate analysis after excluding those with incomplete responses. Multivariate regression analysis was conducted to explore the relationships between socio-demographic characteristics and the levels of agreement of community pharmacist within the three categories, namely safety at workplace, resilience, and support. A p value of less than 0.05 was considered to indicate statistical significance. All analyses were performed with the use of SPSS Statistics version 26 for Windows (IBM Corp., Armonk, NY, USA).

\section{Results}

A total of 217 community pharmacists participated in the current survey. Participants were predominantly females $(74.2 \%)$ and were aged 30 to 39 years $(56.7 \%)$. Nearly all of the respondents were full time pharmacists $(92.6 \%)$ who possessed a bachelor's degree and were either the pharmacist manager or proprietor. Characteristics associated with the study participants were described in Table 1.

\section{Perceptions of community pharmacists on safety, resilience, and support}

A large proportion of participants agreed or strongly agreed that they had access to personal protective equipment to keep themselves safe $(92.2 \%)$ and felt confident that they could remain healthy while working (84.6\%). Participants also felt that during this pandemic, they had been able to balance working with self-care (85.2\%) and kept a positive outlook on their capability to contribute to the society (91.4\%). Most participants felt that they had a good and supportive working relationships with their employer (93.4\%) and were confident in their leadership team's decisions during this period (85.6\%). They also felt that they had sufficient access to the information needed for health and wellness benefits (75.0\%) (Table 2).

\section{Innovations in pharmaceutical services during COVID-19 pandemic}

To ensure the safety of their staff and customers, most community pharmacists modified the environment in their pharmacy to ensure safe passage of customers. These included the installation and creation of some forms of physical barriers at the doorways or in front of the counter $(60.0 \%)$. Some community pharmacies also added markings using tape on the ground to section areas to enable physical distancing between their customers, especially during payment or waiting for pharmacy services (70.4\%). A large proportion of community pharmacies also provided disposable gloves available freely for staff use $(61.5 \%)$, whilst most had also restricted the number of customers that would be allowed to enter the pharmacy $(63.0 \%)$. Three in every four community pharmacies also had freely available hand sanitizers for use by customers (74.1\%) and employees $(81.5 \%)$. Some innovative services which were offered by community pharmacies encompassed medication delivery (39.3\%), remote consultations (31.1\%) as well as curbside delivery (37.0\%) and drivethrough services of medications for their customers (33.3\%). Interestingly, another one in four community pharmacies also started a new collaborative program with the neighboring doctors to refer their patients with chronic disease (Table 3 ).

\section{Response to COVID-19 pandemic}

We also examined how participants baseline characteristics affected their outlook and perception towards responding to COVID-19 crisis. Findings suggested that the level of perceived organizational support was strongly correlated with gender $(\mathrm{r}=0.578, \mathrm{p}<0.01)$, whereas perceived safety of work environment was significantly associated with occupational role of pharmacists $(\mathrm{r}=0.144, \mathrm{p}<0.05)$ (Table 4). Multivariate logistic regression models after adjustment for socio-demographic factors revealed no significant difference with respect to gender, old age, postgraduate education, owner of pharmacy, and full time job in the context of 
perceived workplace safety, resilience, and organizational support during COVID-19 crisis (Table 5).

\section{Discussion}

To our best knowledge, this study is the first cross-sectional study conducted in Malaysia to seek to understand the coping strategies and innovative responses of community pharmacists for COVID-19 pandemic. We found that most community pharmacists were generally well prepared to cope with the pandemic and had a positive outlook towards keeping themselves and their customers safe, thereby further affirming the instrumental role of community pharmacists in implementing public health measures to control the spread of COVID-19. Whilst most community pharmacies had adapted their environment to reduce the risk of transmission, some community pharmacies went further in innovating new services, for instance, providing a drive-through or home delivery service to further minimize contacts among their customers. Interestingly, another key collaboration that was developed was referral services among other community pharmacies as well as with primary care doctors within the vicinity, which had been rarely practiced in Malaysia due to competing commercial interests.

However, we found that less than one in every five community pharmacists in the survey reported to have installed acrylic glass barriers or perspex in front of the counter for the collection of medicine and utilized baskets to receive payments at cashier. This might be due to limited floor space and lack of infrastructure feasibility for applying the physical distancing measures. Notwithstanding, the employees were protected with the complimentary use of gloves and hand sanitizers. Furthermore, there was a growing opportunity for community pharmacists to exploit technologies to facilitate remote consultations, as presently practiced by $31.1 \%$ of the respondents. Despite major investments have been insufficient in health technology industry of low-middle and middle-income countries, the COVID-19 pandemic has propelled the pursuit of convenient and inexpensive telehealth solutions to reduce population movements or physical and social interactions which help to curb the spread of the disease, while maintaining quality of clinical care. To realize a holistic telehealth-enabled future, novel engineering designs, products, and innovations such as smart phones, wearable devices, and instrumented (smart) homes can be accoutered with environmental and biological sensors that are interconnected using $5 \mathrm{G}$ networks to monitor patient health and send messages to assigned clinicians and pharmacists when emergency situations are detected. ${ }^{9-11}$

We observed an overall decrease in level of perception on the dimensions of safety, resilience, and support with increasing age of pharmacists. The majority of participants surveyed $(74.7 \%)$ were mid-career pharmacists aged 30 to 49 years, while only $2.3 \%$ aged 60 years or older. It has been suggested that pharmacists' reactions to work-related conditions and experiences is dependent on age, with middle-age pharmacists expressing less satisfaction compared to both younger and older practitioners. ${ }^{12}$ Our study resonated with a previous observation that pharmacists of 45 to 59 years old were more dissatisfied with their workload and attached more importance of their jobs to patients than did younger or older pharmacists. ${ }^{13}$ We believe that this could be because senior members of the profession had prior experience to coordinate the emergency response to past series of influenza outbreaks that were similarly characterized by novel virus subtypes, specifically SARS outbreak of 2003, H1N1 Influenza of 2009, and New Avian Influenza Viruses H7N9 and H10N8 of 2013. ${ }^{14}$ As such, they could acclimatize better to the policies and work environment for which they have served over numerous years.

Data from this study also found some evidence of gender disparity in organizational support. Albeit a tactful bit of literature found no evidence for gendered differences in ability, the variation in perceptions demonstrates a potential effect of local social norms and cultural influences in achieving supportive organizational culture which is governed by the shared values and beliefs that influence workplace and employee behaviors. ${ }^{15}$ As such, effective strategies involving legislation, allyship, leadership, and professional development of core competencies could transform organizational culture and climate towards gender equality in science, medicine, and global health, hence optimizing health, social, and economic gains. ${ }^{16}$ In many countries, the limited opportunities for hiring, merit, promotion, and access to productive resources of women in the fields of science and medicine warrant policy changes. This is indispensable for addressing and removing barriers to ensure full engagement and participation of women in the workforce, resulting in increased 
satisfaction and more diverse recruitment. ${ }^{15}$

The surprising correlation between perceived workplace safety and occupational role could be plausibly explained by external covariates, including the availability of higher operating budget, business revenue, and staff education programs. Being the owner, partner, or executive officer of a community pharmacy generally has the ultimate responsibility and authority to decide on the company's capital allocation, decision making, and operational management processes. Hence, the extent to which pharmaceutical care of patients and welfare of staff were distributed under the condition of a pandemic would stem from decisions and actions from the corporate boards.

Several limitations of this study should be noted. First, because the study design was cross-sectional, we could not elucidate the causality and temporal patterns between characteristics of community pharmacists and the way they responded to epidemic outbreaks. Second, the study faced challenges to timely repetition and longitudinal follow-up, limiting its utility for long term surveillance to examine the effects of different responses to public health crises on clinical outcomes and general infection control measures. The sample size was small in certain subgroups, particularly among male, those who were older as well as locum pharmacists, which could result in wide confidence intervals and point estimates. Similarly, due to the multiplicity of tests performed, there is a likelihood of occurrence of type 2 errors in the correlations between demographics and the safety, resilience, and organizational support received. As such, we urge caution in the interpretation of the results. In addition, the generalizability of the results might be limited because most of the participants were young women. However, this population is comparatively similar to the general community pharmacist population in Malaysia and many other countries. ${ }^{17-19}$ Our study findings were also restricted to items contained in the survey instrument which was not designed to quantify the magnitude of changes in coping strategies and provision of pharmaceutical services over the pandemic period.

As we did not perform a probability-based sampling method, our results may not be representative of the full diversity of the Malaysian population with respect to race and ethnicity. The questionnaire scope did not measure responses by race or ethnicity of the pharmacists, socioeconomic status of the communities surrounding the pharmacies, geographical locations, and presence of public health programs in the local settings. Nevertheless, we believe that a transdisciplinary, cross-sectoral, coherent approach to infectious disease control should embrace community pharmacies with public health agencies to address the social determinants of health. As such, community pharmacies represent an important infrastructure within the primary healthcare systems for combating the pandemic, contributing a part in patient education, disease surveillance, and dealing with medical supplies to be stockpiled or redirected in an emergency. ${ }^{20}$ The services that can be delivered in community pharmacy settings include medication dispensing for chronic and acute conditions, chronic disease management, recommendations for over-the-counter medications, medication management and adherence support, self-care recommendations, vaccinations, specimen collection, and point-of-care screening or testing services for rapid diagnosis and education of patients about results, lifestyle recommendations, and referral to specialty care if necessary. ${ }^{21-23}$

There may be variations in responses regarding planning and preparedness of community pharmacies to disease outbreaks prior to stringent enforcement of lockdown measures by the authorities since March 18, $2020 .{ }^{24}$ Consequently, the results of this study cannot explicitly refute other ecological drivers of change which may have influenced the findings of this study. It is also noteworthy that the perceptions of pharmacists may change over time in response to social and physical environmental factors. Strict enforcement would improve compliance to rules and standard operating procedures introduced by the government and thus minimizing the risk of community transmission of COVID-19 in pharmacies and public areas. ${ }^{25}$ As compared to face-toface interviews, our study was conducted through online survey which might lead to biased results because it could have dismissed those with high workload, scarce time, and limited connectivity.

The repercussions of lockdown are now being observed in the Malaysian society, where furloughs have turned into redundancies and economic recessions have taken place. As such, we believe it is reasonable to anticipate not only sustained distress and considerable deterioration in supply chain and affordability of long term personal protective equipment along with various businesses and profits downturn, but emergence of 
adverse health impacts of economic recessions due to the precarious job markets and weak social protection systems. The COVID-19 pandemic has engendered numerous daunting challenges for health service provision, especially with differing access to housing, food security as well as social connectivity, all of which are related to public health. Therefore, appropriate and proportionate clinical duties and response to mitigate or manage coronavirus crisis require multidisciplinary high-quality pharmaceutical care from the community pharmacies, alongside sufficiently resourced public health services supported by government policies and programs.

\section{Conclusion}

The findings depict the readiness and integral work performed by the community pharmacists in supporting patients and local communities, particularly the critical role they have played as frontline team members during the COVID-19 pandemic. For societies to control and prevent the transmission of emerging infections, it is essential that public health policies emphasized the need for technical, regulatory, and financial support from the government to improve protection of healthcare workforce and their communities against infectious disease, incorporating community pharmacists into nationally synchronized commitments to responding to any disease outbreak in collaboration with multidisciplinary teams of healthcare providers and specialists.

\section{References}

1. Berenbrok LA, Gabriel N, Coley KC, Hernandez I. Evaluation of frequency of encounters with primary care physicians vs visits to community pharmacies among medicare beneficiaries. JAMA Netw Open. 2020;3(7):e209132.

2. Cadogan CA, Hughes CM. On the frontline against COVID-19: Community pharmacists' contribution during a public health crisis. Res Social Adm Pharm. 2021;17(1):2032-2035.

3. Al Saidi AMO, Nur FA, Al-Mandhari AS, El Rabbat M, Hafeez A, Abubakar A. Decisive leadership is a necessity in the COVID-19 response. Lancet. 2020;396(10247):295-298.

4. Khojah HMJ. Community pharmacy services and preparedness during COVID-19 outbreak in Madinah, Saudi Arabia. Saudi Pharm J. 2020;28(11):1402-1407.

5. Mukattash TL, Jarab AS, Mukattash I, et al. Pharmacists' perception of their role during COVID-19: A qualitative content analysis of posts on Facebook pharmacy groups in Jordan. Pharm Pract (Granada). 2020;18(3):1900.

6. Basheti IA, Nassar R, Barakat M, et al. Pharmacists' readiness to deal with the coronavirus pandemic: Assessing awareness and perception of roles. Res Social Adm Pharm. 2021;17(3):514-522.

7. COVID-19: Guidelines for pharmacists and the pharmacy workforce. The Netherlands: International Pharmaceutical Federation;2020.

8. COVID-19: Resources, statements and initiatives. Malaysia: Malaysian Pharmacists Society;2020.

9. Blandford A, Wesson J, Amalberti R, AlHazme R, Allwihan R. Opportunities and challenges for telehealth within, and beyond, a pandemic. Lancet Glob Health. 2020;8(11):e1364-e1365.

10. Lee JY, Chan CKY, Chua SS, et al. Telemonitoring and team-based management of glycemic control on people with type 2 diabetes: A cluster-randomized controlled trial. J Gen Intern Med. 2020;35(1):87-94.

11. Chakranon P, Lai YK, Tang YW, Choudhary P, Khunti K, Lee SWH. Distal technology interventions in people with diabetes: An umbrella review of multiple health outcomes. Diabet Med. 2020;37(12):19661976.

12. Carvajal MJ, Popovici I. Gender, age, and pharmacists' job satisfaction. Pharm Pract (Granada). 2018;16(4):1396.

13. Carvajal MJ, Popovici I, Hardigan PC. Gender and age variations in pharmacists' job satisfaction in the United States. Pharmacy (Basel). 2019;7(2):46. 
14. Fineberg HV. Pandemic preparedness and response - Lessons from the H1N1 Influenza of 2009. N Engl J Med. 2014;370(14):1335-1342.

15. Coe IR, Wiley R, Bekker L-G. Organisational best practices towards gender equality in science and medicine. Lancet. 2019;393(10171):587-593.

16. Shannon G, Jansen M, Williams K, et al. Gender equality in science, medicine, and global health: Where are we at and why does it matter? Lancet. 2019;393(10171):560-569.

17. Janzen D, Fitzpatrick K, Jensen K, Suveges L. Women in pharmacy: A preliminary study of the attitudes and beliefs of pharmacy students. Can Pharm J (Ott). 2013;146(2):109-116.

18. Anggraini S, Utami W, Zairina E. Factors affecting community pharmacist's service for women with chronic diseases during pregnancy and breastfeeding: Application of the health belief model. $J$ Basic Clin Physiol Pharmacol. 2019;30(6):/j/jbcpp.2019.30.issue-6/jbcpp-2019-0347/jbcpp-2019-0347.xml.

19. Gidman W. Increasing community pharmacy workloads in England: Causes and consequences. Int J Clin Pharm. 2011;33(3):512-520.

20. Gates B. Responding to COVID-19 - A once-in-a-century pandemic? $N$ Engl J Med. 2020;382(18):1677-1679.

21. Lee SWH, Bell JS. Pharmaceutical Care in Asia. In: Alves da Costa F, van Mil JWF, AlvarezRisco A, eds. The Pharmacist Guide to Implementing Pharmaceutical Care. Cham: Springer International Publishing; 2019:191-197.

22. Lee SWH, Mak VSL, Tang YW. Pharmacist services in nursing homes: A systematic review and meta-analysis. Br J Clin Pharmacol. 2019;85(12):2668-2688.

23. Okoro RN. COVID-19 pandemic: The role of community pharmacists in chronic kidney disease management supportive care. Res Social Adm Pharm. 2021;17(1):1925-1928.

24. Shah AUM, Safri SNA, Thevadas R, et al. COVID-19 outbreak in Malaysia: Actions taken by the Malaysian government. Int J Infect Dis. 2020;97:108-116.

25. Sum ZZ, Ow CJW. Community pharmacy response to infection control during COVID-19. A cross-sectional survey. Res Social Adm Pharm. 2021;17(1):1845-1852. 